\title{
Balzac Et La Petite Tailleuse Chinoise, Barbe Bleue et le testament français :de l'intertextualité à le recontextualisation
}

\section{Résumé:}

Dans le présent article, je veux montrer, à travers une lecture intertextuelle de Balzac Et La Petite Tailleuse Chinoise, Barbe Bleue, et le testament français, que le jeu intertextuel auquel se donnent les romanciers est, en plus d'être un mécanisme de re-contextualisation et d'expansion textuelle le plus important à l'intérieur du système littéraire ou sur le plan culturel le plus large, n'a rien d'anodin, dans la mesure où il s'inscrit dans une véritable stratégie discursive et idéologique du romancier qui inaugure par le biais de ce jeu une attitude réflexive et une lecture critique.

Les mots-clefs : l'intertextualité, la recontextualisation, la puissance transculturelle et mémorielle de la littérature, les stratégies narratives.

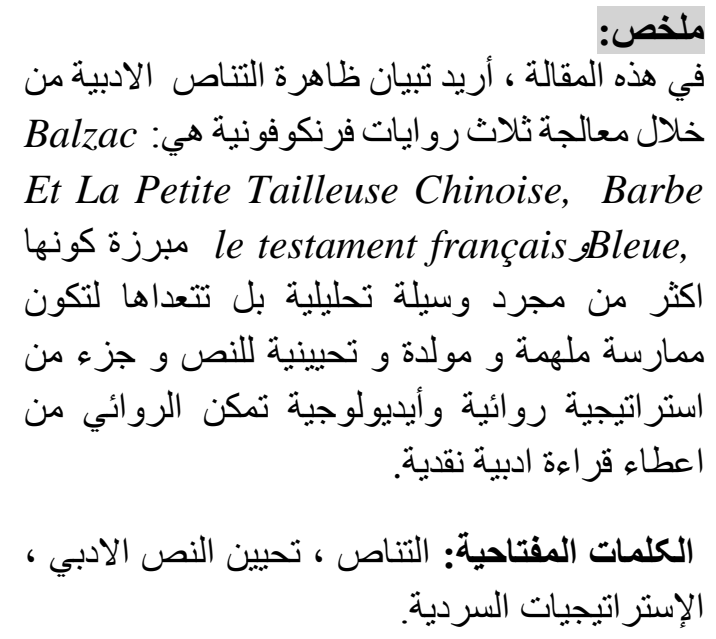

\section{Fatima TABDJOUNE}

Département des lettres et langue française Université des frères Mentouri Constantine 1

\begin{abstract}
Introduction:
Balzac et La Petite tailleuse chinoise $^{(1)}$, Barbe Bleue ${ }^{(2)}$ et Le testament français $^{(3)}$ sont des œuvres francophones au goût de la littérature française. L'héritage culturel et littéraire français est le point de rencontre de ces romans par-delà la multiple appartenance culturelle, linguistique et géographique des écrivains.
\end{abstract}


Essentiellement de construction intertextuelle complexe ces romans font appel à l'intertextualité qui ne se limite pas à un outil d'analyse littéraire mais qui s'avère comme une pratique inspiratrice et actualisante de l'écriture littéraire. Dai Sijie fait référence, à l'aide de la technique de la mise en abyme, à Honoré De Balzac et à la lointaine France, les appelle carrément à la rescousse afin de juguler sa haine et survivre à la politique destructrice de la Chine sous le règne de l'empereur Mao Zedong durant les années 70. Amélie Nothomb réécrit et remet au goût du jour, sous forme d'un roman dialogué, une œuvre ancienne, un conte populaire de Charles Perrault datant du XVII ${ }^{\text {ème }}$ siècle et elle donne à lire une réflexion très profonde sur l'amour, sur les relations conjugales et notamment sur la condition de la femme au XX ${ }^{\text {ème }}$ siècle. André Makine, quant à lui, prisonnier d'une langue et d'une mémoire nostalgique et mélancolique grand-maternelle, il s'invente des pères : les grands classiques de la littérature française tel Proust, Rimbaud, Badelaire, Hugo, Nerval, Gautier. Un monde livresque qui a nourri l'imaginaire d'un enfant russe au fond de la Sibérie où il a vécu les horreurs de la politique menée par la Russie Soviétique.

Une lecture intertextuelle de ces romans nous dévoile un véritable syncrétisme des temps et des lieux, un télescopage de textes, de langues et de cultures. Ces romans s'inscrivent dans une complexité de rapports entres des éléments constamment en interaction, ces rapports raisonnent comme une jonction et une disjonction de l'écrivain et de son œuvre car si le romancier est chinois, belge ou russe, l'œuvre, elle, est de langue et de culture françaises. Cette langue de passion représente pour ces écrivains une langue de refuge, une terre d'accueil et un foyer culturel.

A l'aide de l'intertextualité, qui est née dans une multiplicité d'origines et d'approches fondées par des théoriciens venus d'horizons très divers et d'une multiplicité de voix divergentes mais aussi complémentaires. Dai Sijie, Amélie Nothomb et André Makine ont réussi à créer un espace littéraire francophone riche, nourri de différentes cultures, un lieu de rencontre d'une multiplicité de textes, de voix et d'identités. Cette pratique littéraire permet, non seulement, d'enrichir le texte abouti mais de l'inscrire, d'une part, dans l'espace autoréflexif et auto représentationnel du langage littéraire, et d'autre part, elle permet de rappeler la puissance transculturelle et la dimension mémorielle de cette littérature universelle qui abolit les frontières spatio-temporelles, culturelles et linguistiques.

La dimension mémorielle de la littérature nous rappelle la mémoire mélancolique de Tiphaine Samoyault ${ }^{(4)}$ : «tout est dit, et l'on vient trop tard, depuis plus de sept mille ans qu'il y a des hommes, et qui pensent ». Dai Sijie, Amélie Nothomb et André Makine, ont relevé le défit de se servir de " tout est dit » pour parler, paradoxalement, de soi ou d'une collectivité avec les mots d'un autre. Grâce à l'esprit créatif de chaque romancier, le passage d'un système de signifiant (l'hypotexte) à un autre (l'hypertexte) ne s'effectue pas sans 


\section{Balzac Et La Petite Tailleuse Chinoise, Barbe Bleue et le testament français :de l'intertextualité à le recontextualisation}

créativité ni inventivité, il exige une nouvelle articulation de la positionnalité énonciative et dénotative du romancier, autrement dit, une nouvelle contextualisation aussi bien sémantique que temporelle. Cette juxtaposition de différents objets culturels, littéraires et structures sémantiques permet de reproposer le paratexte dans un nouveau contexte, pour produire intelligemment un autre sens à travers une narration inventive, plus complexe mais surtout plus actuelle, il s'agit bien d'une recontextualisation.

L'objectivation symbolique du projet de ces écrivains et leurs idéologies se réalisent en sortant de l'unité de signification qui est le texte lui même et en s'intéressant à l'intertextualité non pas uniquement comme phénomène littéraire mais comme un signe vers le monde et cela à travers la réutilisation d'un autre texte considéré comme un premier élément significatif et interprétatif, mais surtout une toile de fond, à partir de laquelle s'exprime et se réinvente le sens du deuxième texte dans un mouvement et une osmose entre saisie et adoption, reproduction et création, et réalité et imagination. Ainsi la recontextualisation est assurée grâce à diverses stratégies narratives mises en place par chaque écrivain, dans un contexte de réception et d'interprétation plus actuelle.

\section{1-L'intertextualité du structuralisme à la théorie de la réception}

La notion d'intertextualité est née à la fin des années 60 , mais son origine remonte bien avant avec les travaux de $\mathrm{M}$. Bakhtine et plus précisément avec le principe du dialogisme et accessoirement avec le terme de polyphonie. À travers l'analyse de l'œuvre de Dostö̈evski (5), Bakhtine élabore sa théorie de dialogisation interne du discours qui postule que tout énoncé d'un locuteur, par sa dimension linguistique, est habité de discours antérieurs et renvoie à d'autres textes: «le langage n'est pas un milieu neutre. Il ne devient pas aisément, librement, la propriété du locuteur. Il est peuplé et surpeuplé d'intentions étrangères. Les dominer, les soumettre à ses intentions, c'est un principe ardu et complexe $\gg^{(6)}$

A la fin des années 60 , l'intertextualité commence à s'inscrire dans une théorie du texte à plusieurs facettes qui insiste sur la signifiance et la productivité de l'écriture. J. Kristeva traduit les écrits de Bakhtine et diffuse ses idées de façon marquante en France. Elle accrédite la terminologie de «l'intertextualité » en $1966^{(7)}$ dans le cadre de ses travaux de présentation des théories Bakhtiniennes du dialogisme et de la polyphonie dans le discours romanesque. En s'inspirant du concept de dialogisme, elle pose les fondements de l'intertextualité pour désigner l'effet textuel ; l'interaction et le croisement des textes dont la cause est le dialogisme du discours littéraire : "le mot(le texte) est un croisement de mots (de textes) où on lit au moins un autre mot (texte) ${ }^{(8)}$

Kristeva définit le texte comme un appareil translinguistique, qui a pour fonction la redistribution des signifiants, il les transpose, les juxtapose et les transforme. Le langage est en ce sens productif. C'est dans cette transformation des 
signifiants que se produit le sens du texte. Les rapports entre ces différents signifiants sont des rapports intertextuels : "Tout texte se construit comme une mosaïque de citations, tout texte est absorption et transformation d'un autre texte. A la place de la notion d'intersubjectivité s'installe celle d'intertextualité, et le langage poétique se lit, au moins comme double ${ }^{(9)}$

Cette mosaïque est le résultat du processus transformateur des rapports entre ces signifiants. Dans l'intertextualité, Kristeva voit le principe de la littérarité, la spécificité du texte littéraire ; à cela s'ajoute la dimension polysémique du texte littéraire. Pour Kristeva, il n'y a pas de littérature sans l'intertextualité, et il n'y a pas d'intertextualité sans transformation d'énoncés, sans mouvement du langage dénotatif vers un langage connotatif.

On reconnait à $T$. Todorov le mérite d'instaurer un rapport quasi-équivalent entre le dialogisme et l'intertextualité : «le caractère le plus important de l'énoncé, ou en tous les cas le plus ignoré est son dialogisme, c'est-à-dire sa dimension intertextuelle $»^{(10)}$

M. Riffaterre explore depuis la fin des années70, sa théorie de la réception en partant de l'intertextualité de Kristeva et en y rajoutant la notion de l'acte de la lecture. Il définit l'intertextualité comme un mode de perception du texte : la signification intertextuelle est atteinte par le biais de la reconnaissance de l'intertexte, elle est programmée par ce dernier : "L'intertextualité est un phénomène qui oriente la lecture et qui gouverne éventuellement l'interprétation ${ }^{(11)}$.

Les rapports étudiés par Riffaterre sont de l'ordre des microstructures sémanticostylistiques (au niveau de la phrase, du fragment, ou du texte). Pour sa part, il définit l'intertextualité comme : «La perception, par le lecteur, de rapports entre une ouvre et d'autres, qui l'ont précédée ou suivie. Ces autres œuvres constituent l'intertexte de la première. La perception de ces rapports est donc une des composantes fondamentales de la littérarité d'une cuvre $»^{(12)}$

Les divers phénomènes intertextuels ont commencé, depuis le début des années 80 , à faire l'objet d'une description à visée poéticienne notamment avec la parution de Palimpsestes, en 1982 de G. Genette, qui rebaptise l'intertextualité d'un nom plus général et plus englobant qui est «la transtextualité » ou la transcendance du texte qui est " tout ce qui met (le texte) en relation manifeste ou secret avec d'autres textes ${ }^{(13)}$. Cette relation désigne un ensemble de cinq catégories opératoires exhaustives qui englobent l'intertextualité Kristevienne, au sens strict, résumée comme étant «la présence littérale (...) d'un texte dans un autre ${ }^{(14)}$.

2-Intertextualité, recontextualisation et syncrétisme des temps et des lieux "Tout texte est un intertexte; d'autres textes sont présents en lui, à des niveaux variables, sous des formes plus ou moins reconnaissables: les textes de la culture antérieure, ceux de la culture environnante ; tout texte est un tissu nouveau de citations révolues. Passent dans le texte, 


\section{Balzac Et La Petite Tailleuse Chinoise, Barbe Bleue et le testament français :de l'intertextualité à le recontextualisation}

redistribués en lui, des morceaux de codes, des formules, des modèles rythmiques, des fragments de langage sociaux, etc., car il y a toujours du langage avant le texte et autour de lui.» ${ }^{(15)}$

Apres un bref aperçu des différentes théorisations de l'intertextualité, notre outil d'analyse, et son évolution à travers le temps et sans vouloir se perdre dans un foisonnement terminologique qui entoure cette notion, nous avons remarqué que le principe de dialogisme et d'interaction entre les textes est en filigrane chez tous les théoriciens, qui, soulignent chacun selon sa théorie , que la perception des rapports intertextuels entre les textes est une composante fondamentale de la littérarité et de la textualité d'une œuvre littéraire.

A travers l'analyse des œuvres du corpus, nous allons remarquer que la pratique intertextuelle est une pratique inspiratrice, féconde et actualisante du texte et de l'écriture littéraire, en effet, elle assure sa recontextualisation.

L'intertextualité se révèle comme une pratique constitutive du texte littéraire, les romans se nourrissent des textes d'autrui qu'ils absorbent puis transforment. Elle est en plus d'être un outil d'analyse, une clé de la lecture et de l'écriture littéraire, ainsi conçue par R. Barthes en introduisant la notion de productivité : «Tout texte est un intertexte, une productivité, non seulement parce qu'il s'élabore à partir d'autres textes, assimilés et transformés, refus et interprétés mais parce qu'il a besoin du lecteur pour accéder à la signification ${ }^{(16)}$.

\section{2-1Balzac Et La Petite Tailleuse chinoise : fiction, révélation et contestation} politique

"La mémoire culturelle fait coexister des éléments qui sont divers à beaucoup d'égards, et divers aussi par leur âge. Ils sont présents ensemble, puisqu'ils coexistent dans l'actualité de la mémoire, mais ils ne proviennent pas tous du même passé » ${ }^{(17)}$.

Balzac et La Petite Tailleuse Chinoise, est une œuvre si simple en apparence, qui relève le challenge de dire tant de choses à la fois. Dai Sijie avait compris, que l'écriture littéraire est la seule activité durable, qui lui permettrait de survivre à l'empereur Mao et à sa politique égocentrique et destructrice pendant la révolution culturelle chinoise des années 1970. Des textes littéraires sont plantés et constituent le décor fictif pour accueillir et amoindrir, atténuer quelques peu les durs sévices historiques.

Durant la politique de la rééducation chinoise conduite par Mao est ses idéologues aigris, ce qui a été sans doute le plus bafoué, ce sont incontestablement les productions intellectuelles, particulièrement la littérature. Dai Sijie entreprend de la réhabiliter, non pas à travers le développement d'un ouvrage théorique ou une œuvre contestataire virulente qui alignerait des chiffres et des statistiques, mais calmement, au moyen d'un récit des plus simples, parce que partiellement authentique. Ainsi l'auteur fait appel à la littérature, dont il a 
été sevré, pour reconquérir cette liberté perdue, et suggérer la puissance transculturelle et mémorielle de cette vénérable discipline.

Mao appréhendait, certainement, les intellectuels: cela s'explique par l'interdiction des enseignements dans les écoles et les universités et les séances d'autodafé qu'il organisait. Mais pourquoi interdire des œuvres littéraires de fiction qui sembleraient inoffensives ?

C'est certainement parce que la fiction est essentiellement une forme de liberté où tout est permis et réalisable, c'est probablement, selon T. Todorov, la littérature est « par ce que celle-ci fait, plutôt que par ce qu'elle est ${ }^{(18)}$. Cela déplait fort au régime totalitaire.

Les romans sont moins inoffensifs qu'ils ont l'air, parce que une histoire racontée, à travers la proposition d'un autre monde, offre une autre possibilité, celle de la reconstitution d'un soi, celui du lecteur. Paul Ricœur définit la lecture comme «la refiguration de l'action par le destinataire du récit, la réappropriation du sens $d u$ récit ${ }^{(19)}$, il confirme que " contre le moi maitre de lui-même, le soi disciple de l'autre ${ }^{(20)}$. N'est -il pas le cas de la Petite Tailleuse qui symbolise ce mouvement, cet aller-retour entre l'apprentissage d'un roman et l'apprentissage du monde ?

Le régime dictatorial de Mao ne craignait pas l'affrontement avec l'occident mais il avait plutôt peur de l'influence et du dialogue des esprits. Les deux protagonistes Luo et le Narrateur convoitent une valise pleine de livres rêvés et interdits : "les grands écrivains nous accueillirent à bras ouverts : à leur tête se tenait notre vieil ami Balzac avec cinq ou six romans, suivis de Victor Hugo, Stendhal, Dumas, Baudelaire, Romain Rolland, Rousseau, Tolstö̈, Gogol, Dostoïevski, et quelques écrivains Anglais : Dickens, Kipling, Emily Brontë $»^{(21)}$. Mao avait de quoi avoir peur! Luo avoue que «de les toucher du bout de doigts, il me semblait que mes mains devenues pâles, étaient en contact avec des vies humaines ${ }^{(22)}$. En posant sa main sur la mallette, comme pour prêter serment, ce jeune émancipateur déclare que : "avec cette valise, je vais transformer la Petite Tailleuse, elle ne sera jamais une simple montagnarde ${ }^{(23)}$.

La Petite Tailleuse chinoise belle mais inculte sera rééduquée par Luo grâce à la lecture d'un « livre mine et usé » ${ }^{(24)}$, Ursule Mirouët (1841), un médium qui a la force de franchir toutes les frontières spatiales et temporelles, et assure un va et vient entre fictionnel et le réel. L'histoire de la vie et de l'amour, une histoire qui paraît aussi vraie que celle de leurs voisins, au point que Luo se sent chez lui dans la maison que la jeune héroïne occupa à Nemours, en passant par les illusions perdues(1837), Eugénie Grandet (1833), Cousin Pont (1847) et enfin le chef-d'œuvre de Balzac Le Père Goriot (1835), l'œuvre la plus représentative du roman d'apprentissage. Le pouvoir de mots effrayait Mao, lui, qui visait, durant les années 1970, à produire des individus qui soient incapables de penser en dehors des références communistes. 


\section{Balzac Et La Petite Tailleuse Chinoise, Barbe Bleue et le testament français :de l'intertextualité à le recontextualisation}

Le narrateur ne se contente pas de faire référence à une œuvre externe à son histoire, mais il finit par établir des liens, voire une similitude. Lorsqu'il parle du premier des quatre volumes de Jean Christophe, il s'est approprié de la fiction et lui a donné un sens par rapport à son histoire. À travers la technique de la mise en abyme, c'est la dimension référentielle de l'histoire racontée qui est dédoublée dans le récit réflexif: «Tout miroir interne réfléchissant l'ensemble du récit par réduplication simple, répétée ou spécieuse. ${ }^{(25)}$. Le narrateur considère le livre de Jean Christophe comme un parfait enseignant, voire un magicien «une fois que je l'avais fini, ni votre sacrée vie, ni votre sacré monde n'étaient plus même qu'avant(...) ma pauvre tête éduquée et rééduquée l'ignorait » ${ }^{(26)}$.

Luo raconte au père de La Petite Tailleuse le compte de Monte-Cristo, ce vieux tailleur devient le disciple de Dumas et l'influence du romancier français apparait dans les nouveaux modèles de vêtements confectionnés et notamment ceux destinés aux marins: "les vareuses à épaules tombantes et à grand col carré en arrière et pointu en avant(...) les pantalons bleus de matelots avec les pattes larges et flottantes ${ }^{(27)}$. Ces vêtements faisaient sentir l'odeur de la méditerranée, émaner le parfum de la côte d'azur, il a même crée un motif: une sorte d'ancre à cinq becs.

Dai Sijie marque avec Balzac et La Petite Tailleuse Chinoise, un roman d'un abord assez facile, d'une facture sobre et d'un débit fluide et coulant la littérature des cicatrices ${ }^{(28)}$, car cette œuvre est, avant tout, le témoignage d'une victime porte-parole de millions d'autres victimes, silencieuses, d'un moment de folie collective sans précédant dans l'histoire moderne. La littérature des cicatrices a révélé le challenge de milliers de jeunes intellectuels qui se souvenaient ou qui pourraient se souvenir de cette haute période totalitaire dans un contexte d'oppression masquée: "Les nouveaux maîtres du pouvoir étaient de vieux communistes, parfois même des maö̈stes d'origine: pas question, pour eux, de changer ni le régime ni la définition d'une histoire engendrée par le Parti. Ils tranchèrent donc rapidement : en Mao Zedong, le stratège politico-militaire de la révolution devait être admiré, et le fondateur du régime approuvé(...), le jugement historique restait donc favorable, non seulement à Mao, mais plus encore au régime communiste » (29)

Les personnages de Dai Sijie incarnent ses idées sur l'amour et l'humanisme, à travers lesquels ils abordent la condition de la femme chinoise et son émancipation. Nous relevons clairement le thème principal de la littérature des cicatrices qui est celui de l'amour et de la foi (30): l'amour humain est la seule solution pour résoudre les problèmes sociaux ${ }^{(31)}$. Le narrateur et Luo ont saisi cette force de nuance des mots, tout comme la Petite Tailleuse, pourtant simple villageoise de son état (en dépit du statut privilégié de son père appartenant pratiquement à l'aristocratie, orpheline de mère et échappant à la férule de son père, souvent en déplacement), celle-ci devrait être pour l'auteur la jeune fille 
idéale prête à succomber à la voix de l'émancipation. Quoi de plus émancipatrice assurément que la littérature et l'amour d'un jeune homme audacieux, débrouillard et très éloquent, sans compter les soins et l'adoration platonique du narrateur: "elle m'a dit que Balzac lui a fait comprendre que la beauté d'une femme est un trésor qui n'a pas de prix $\gg^{(32)}$.

Luo a négligé la véritable puissance et l'influence de la culture qui a transformé sa bien-aimée en une héroïne balzacienne. Les mots échappent à tout consensus, à travers une apposition d'un décor chinois à la lointaine France, à sa culture et sa littérature, Dai Sijie conteste et révèle une politique destructrice et humiliante de la Chine des années 1970. Mais quel est l'enjeu d'Amélie Nothomb qui recrée et actualise une œuvre ancienne de la littérature française ?

\section{2-2 Barbe Bleue : texte et prétexte}

" À la fois parce que la littérature est transmission, mais aussi parce qu'elle appelle la reprise, l'adaptation d'un même propos à un public différent. Et de la même manière qu'un nouvel amour fait naitre le souvenir de l'ancien, la littérature nouvelle fait naitre le souvenir de la littérature » ${ }^{(33)}$

A travers la réécriture littéraire qui est, selon Georges Molinié, "une activité scripturaire qui s'établit entre deux éléments. L'un de ces éléments est évidemment stable, qu'il s'agisse d'un discours littéraire de base, réalisé sous la forme d'un texte, ou de tout un style. L'autre élément peut être présenté comme l'écriture d'un nouveau texte, ou la mise en exercice d'un nouveau style ${ }^{(34)}$, Nothomb remet au goût du jour, La Barbe Bleue, une œuvre ancienne qui date du XVII ${ }^{\text {ème }}$ siècle, l'un des contes de Charles Perrault qui est «sont faussement populaires, faussement destinés aux enfants, ces contes sont écrits par un lettré pour un public d'autres lettrés, capables de goûter l'humour cruel d'histoires dont la popularité auprès des enfants ne s'est faite la plupart du temps qu'au prix de lectures qui ignorent ou modifient leur fin ${ }^{(35)}$.

Amélie Nothomb s'efforce de reprendre consciemment un modèle, un texte littéraire, un hypotexte, pour le transformer, selon ses intentions diverses, en variant et le degré de proximité avec le texte original et les modalités d'écriture. Nous assistons à un savant mélange entre création et imitation. C'est tout un mouvement d'appropriation, d'interprétation, de transformation, d'imitation et de transgression.

Nothomb a réécrit le conte de Perrault sous forme d'un roman dialogué, les discussions entre les protagonistes sont centrées sur la confiance et le jeu du désir et de l'interdit. La romancière a repris le héros de Perrault, La Barbe Bleue, tueur de femmes, un personnage laid d'une réputation suspecte mais qui attire par ce qu'il possède et l'étrangeté de son visage les femmes curieuses. Nothomb le réhabilite, La Barbe Bleue nothombienne tue toujours mais il est policé, âgé de 41 ans, elle lui donne un nom extravagant Don Elemirion Nibal Y Miclar, un 


\section{Balzac Et La Petite Tailleuse Chinoise, Barbe Bleue et le testament français :de l'intertextualité à le recontextualisation}

personnage entouré de mystères, un homme orgueilleux, un fou amoureux, un grand inquisiteur, un des grands aristocrates d'Espagne, sa famille descend des Carthaginois et du Christ. Un homme d'or et d'argent plongé dans la dépression, retiré volontairement, dans une demeure féérique, du monde extérieur qu'il juge vulgaire et ennuyeux.

La vie de Don Elemirion Nibal Y Miclar tourne autour de l'amour, du goût des couleurs, de l'interdit, de la confiance et d'un secret à respecter. Il tombe amoureux de l'une de ses locataires : une jeune professeur belge à l'école de Louvre, âgée de 21 ans, Saturnine Puissant, la voix des femmes refroidies, elle semble la locataire idéale, une femme unique, originaire, ayant du goût et une intelligence en couleur, celle qui partagera son désir et respectera son secret.

Dans Barbe Bleue, il est moins question du secret de la disparition des huit femmes locataires que de la cause de leur disparition qui est celle de la curiosité envers un interdit: "Le seul crime de ces 8 femmes était la curiosité envers un homme» ${ }^{(36)}$, mais elles oublient que «tout droit implique une sanction en cas d'infraction $\gg^{(37)}$.

Le désir de savoir, d'ouvrir les portes, de se servir des clefs, de regarder, de violer l'interdit, d'agresser et de ne pas respecter la particularité et le secret d'autrui, Don Elemirio s'est servi de ces défauts pour entrainer ses huit bien-aimées dans la chambre noire, un endroit au froid mortel parce qu'elles n'ont pas respecté sa singularité : «il n'est pas un fou mais un homme épris d'absolu, confronté 9 fois à une question terrible : quelle est la juste frontière entre l'aimée et soi ? ${ }^{(38)}$ La construction intertextuelle, notamment, par le biais de la réécriture du roman, dote l'univers romanesque d'une dynamique textuelle, le lieu privilégié de la rencontre et l'interaction entre les textes et les fragments de textes.

Depuis 21 ans, Don Elimirion est cloîtré dans une demeure magique, avec ses lectures de la bible, de l'inquisition et de romans: "ses vieux livres suffisent pour exister ${ }^{(39)}$. Ces livres lui permettent une ouverture vers une autre vie possible à laquelle Don Elemirio se confond et confond le sort de ses femmes bien-aimées à celui des personnages romanesques et hérö̈nes de ses lectures, il prend ses lectures pour la réalité. Nous assistons à un mouvement dialectique entre cet univers romanesque et fictionnel et la réalité fictive du roman, parce que « Le désir de la littérature est d'être littérature » ${ }^{(40)}$.

Il rend hommage à un compatriote noble aussi catholique et aristocrate que lui : "le Christ a le comportement le plus espagnol au monde. C'est Don Quichotte, en mieux. Et vous ne nierez pas que Quichotte est archiespagnol ». ${ }^{(41)}$. Il confond Saturnine à Dulcinée, l'inspiratrice imaginaire de Don Quichotte mais qui était, malheureusement, la cause de son échec puisque Dulcinée réduit à néant toute la crédibilité du chevalier. Don Elemirio savait- il que chercher une Dulcinée c'est plutôt chercher une chimère, une dévotion et un amour sans fin? 
Perrault réapparait avec le conte de Peau d'Âne(42), ce conte aborde un sujet tabou: l'union incestueuse et la violation d'un interdit. Don Elemirio est à l'image du protagoniste de l'histoire, ce roi de conte de fée, il s'identifie et se confond à ce personnage qui était prêt à tout sacrifier jusqu'à la seule source de sa richesse pour satisfaire son envie. Don Elemirio ne serait-il pas ce roi, généreux qui a tout partagé avec ses neuf femmes colocataires, animé par un désir inexpliqué, cet homme occidental qui ne peut pas se voir trahi, désobéi et non respecté ?

Plus loin ${ }^{(43)}$, Elemirio se révèle en bon lecteur des classiques français, il convoque le roman de Mme de La Fayette, La princesse de Clèves ${ }^{(44)}$, le premier roman de la littérature française d'analyse des cœurs qui accorde à l'héroïne une psychologie complexe ${ }^{(45)}$. L'histoire tourne autour, principalement, d'une envie illégitime, de la jalousie, la trahison et la lutte intérieure entre l'amour conjugal et l'amour-passion.

Il rapproche Saturnine à la princesse de Clèves : «vous n'êtes pas si différente, à votre manière, de la princesse de Clèves » ${ }^{(46)}$ à Melle Chartres qui incarne la perfection par sa beauté physique, sa vertu et sa noblesse, une jeune demoiselle inexpérimentée à la vie mondaine qui n'a aucune expérience de l'amour, elle se marie avec le prince de Clèves un homme aristocrate, noble, honnête et d'une grande droiture morale mais avec qui elle ne partage pas son amour et tombe amoureuse de duc de Nemours.

L'influence de la lecture a entrainé Don Elemirion à choisir le sort de chacune des femmes locataires en l'identifiant aux personnages fictifs avant de les entrainer intelligemment à la mort, à l'exception de Saturnine qui, grâce à sa prudence, elle a échappé au fantasme du propriétaire, elle a réussi à rester cette Dulcinée tant désirée, inaccessible et imaginaire, une princesse de Clèves qui n'a pas cédé à la tentation de la trahison, une peau d'âne qui a repoussé ses peurs, et a respecté l'interdit.

Pour conclure, Barbe Bleue serait-il uniquement une réécriture d'un conte de fée, ou un prétexte pour convoquer ce monde romanesque de conte de fée dans lequel on assiste à une belle mosaïque, une réalité fictive qui s'inspire d'un univers fictionnel plus vaste?.

Dans Barbe Bleue, Il s'agit plutôt d'apprécier ce rapport paradoxal à plusieurs niveaux entre l'hypotexte et l'hypertexte, une relation de proximité et de distance, de reproduction et d'écart, de copie et d'invention.

L'originalité de l'œuvre de Nothomb, est dans l'assomption de la mémoire littéraire et le dépassement par l'appropriation et la disposition nouvelle d'une œuvre qui lui est antérieure, ce jeu, cette dimension de l'auto-référentialité de la littérature témoigne du génie de l'écrivaine à réhabiliter du déjà-dit.

Il n'y a pas de soumission absolue à l'hypotexte, Nothomb réécrit le conte de La Barbe Bleue dans une logique d'argumentation qui lui a permis d'aborder une thèse, sous un angle nouveau. Elle a intériorisé son modèle d'inspiration, pour 


\section{Balzac Et La Petite Tailleuse Chinoise, Barbe Bleue et le testament français :de l'intertextualité à le recontextualisation}

lui rendre hommage, lui redonner sa richesse, et créer une dynamique qui articule l'ancien et le nouveau texte, dans le but de permettre une ouverture et une continuité. «J'ai imaginé, je me suis ressouvenu et j'ai continué» ${ }^{(47)}$.

Perrault en intitulant le conte : La Barbe Bleue a réussi selon, l'une de ses stratégies narratives et discursives, à déplacer le regard vers une apparence physique étrange qui est la couleur bleue de la barbe du héros, pour dissimuler toute une moralité qui se cache derrière le conte, un caractère qui a frappé de stupeur les personnages du conte ainsi que les nombreuses réécritures qui reprennent et interprétant la couleur bleue ${ }^{(48)}$, alors que Nothomb a échappé à cette tentation et nous offre à lire un roman aux couleurs du spectre de la lumière

Longuement analysé, dans son ouvrage, psychanalyse des contes de fées ${ }^{(49)}$, Bruno Bettelheim dévoile le secret de la Barbe Bleue et résume l'histoire en une interaction entre deux sentiments : l'amour jaloux et les sentiments sexuels dans un couple mal assorti selon l'expression de Florence Fix ${ }^{(50)}$. Il analyse de plus près les éléments constitutifs du conte à savoir : la clef, le sang, la fête, l'absence de la Barbe Bleue, le secret et la chambre interdite pour conclure que ce conte est relatif à la tentation sexuelle, il illustre le comportement d'un mari jaloux et violent porté par sa colère et qui croit faire justice, châtie cruellement par la peine de mort ses femmes, une sanction qui a toujours suggéré l'infidélité conjugale. Nothomb fait appel à cette histoire du temps arrêté, à l'image du couple déséquilibré et donne à lire une réflexion sur l'amour et la mort pour « interroger l'intimité, la confiance et l'interdit dans le couple occidental moderne » ${ }^{(51)}$, un couple souvent mal assorti, incapable d'évoluer, de se comprendre, de se pardonner, il est moins question de mariage d'amour et d'affinités mais une relation qui unit un mari rigide, jaloux d'un lourd passé opaque, à une épouse curieuse mise à l'épreuve de la loyauté, de l'obéissance, de la fidélité et de la trahison qui a tenté de percer le secret masculin et qui cède à la tentation sexuelle, alors que ,paradoxalement, rien de plus humain que de succomber à la tentation. L'aspect le plus significatif du conte, qui mélange crime et sexualité( ${ }^{(52)}$, auquel Nothomb fait intelligemment et discrètement allusion est celui du pardon: l'infidélité conjugale doit être pardonnée parce que le partenaire trahi en souffrira lui-même, cet homme mésestimé et violent, collectionneur de mariage dont les expériences nombreuses, n'est qu'un homme malheureux, qui souffre d'une crise d'identité masculine de sur virilité et de puissance.

Barbe Bleue, en effet, consiste à «dire autre chose semblablement ${ }^{(53)}$, cette écrivaine se révèle une féministe bien engagée qui défend la femme, son identité, sa détresse et le manque de confiance à son égard qui pousse à l'extrême sa curiosité quand on la prive de partage et on lui cache un secret car : " la force de tout secret réside avant tout dans le fait même qu'il y ait secret : 
dans l'angoisse et dans l'humiliation d'en être exclu, corrélativement, dans le désir passionné d'y être, d' en être », d'être comme on dit dans le secret. " ${ }^{(54)}$ Tout comme Dai Sijie et Amélie Nothomb, André Makine se ressource des textes d'autrui, ils suggèrent son imaginaire, guident ses souvenirs et ses inspirations pour permettre la naissance d'un monde fabuleux ancré dans une réalité moins agréable à relater.

\section{2-3.Le testament français : entre le rêve et la réalité}

"Tout être est composé de vies et de rencontres multiples, un fourmillement avec ses tentions, ses aspirations contraires, une mémoire mouvante, un shaker ambulant qui tente d'amalgamer toutes ces influences pour en tirer les saveurs propres à son existence » ${ }^{(55)}$

Le testament français, est un roman de l'aveu même de son auteur à inspiration autobiographique, il s'agit d'une intrigue complexe et d'une narration circulaire et linéaire à la fois, qui retrace la vie d'un enfant jusqu'à l'âge adulte, un personnage en pleine émancipation et reconstruction de son unité de personne, quand tout s'offre à lui en double. Ce roman s'inscrit dans un héritage culturel et littéraire français : André Makine, cet écrivain d'origine russe, a quitté son pays natal en 1980 pour s'installer en France où il publie ses premiers romans écrits directement en français, sous un pseudonyme en tant que traducteur. Ce romancier est devenu «un co-auteur de sa vie quant au sens » ${ }^{(56)}$, son œuvre est le témoignage d'un véritable dédoublement, à plusieurs niveaux, entre notamment une appartenance désirée à la France, et une origine russe bien défendue: «la Russie peut-être cruelle, atroce... mais elle n'est jamais petite ${ }^{(57)}$.

Pour fuir aux automatismes de sa langue maternelle, le russe, Makine se réinvente dans sa langue grand-maternelle, qui possède une puissante mémoire littéraire, celle d'une vielle Atlantide perdue: la langue française «qui modelait les hommes, sculptait les objets, ruisselait en vers, rugissait dans les rues envahies par les foules, faisait sourire une tsarine venue du bout du monde...Mais surtout, elle palpitait en nous, telle une greffe fabuleuse dans nos cœurs, couverte déjà de feuilles et de fleurs, portant en elle le fruit de toute une civilisation. ${ }^{(58)}$. Cette langue refuge est une «mystérieuse matière invisible et omniprésente ${ }^{(59)}$, objet de transmission par une mémoire sélective, nostalgique et mélancolique de sa grand -mère Charlotte, une française à la quête de monde qui n'existe plus. Le français est devenu, plus tard, pour cet enfant une langue de passion et une terre d'accueil.

La France héritière de la beauté du français, langue de liberté, de rêve et d'imagination, ce pays est convoqué par sa littérature, le héros s'invente pour pères des grands classiques littéraires et initiateurs à l'amour, à l'élégance, à la mélancolie et à la passion : Proust, Rimbaud, Baudelaire, Hugo, Nerval, Musset, Gautier, Corneille, Rabelais, Balzac, Sand,... un monde livresque pleins 


\section{Balzac Et La Petite Tailleuse Chinoise, Barbe Bleue et le testament français :de l'intertextualité à le recontextualisation}

d'artifices. Cet univers englouti par le temps est convoqué par des fragments de textes grâce au talent de la conteuse Charlotte ; une grand-mère inspiratrice et émancipatrice : «chaque soir ressemblait à un fabuleux matras d'alchimiste où s'opérait une étonnante transmutation du passé. Les éléments de cette magie étaient pour nous moins mystérieux que les composantes de la pierre philosophale ${ }^{(60)}$. Le personnage principal Aliocha grandit dans un univers qui se situe à la frontière de l'imagination et de la réalité atroce de la Russie soviétique, une société très difficile et soumise à la tyrannie et à l'oppression. S'exprimer en langue française est également une dissidence esthétique, ce choix est considéré comme une non aliénation au système soviétique et ses valeurs.

A travers cette construction intertextuelle, Makine dévoile et fictionnalise ce que la Russie de l'époque soviétique trie, organise ou oublie. L'histoire, semble, lui peser comme un destin, elle est en filigrane dans toute ou presque sa production littéraire : "Renouer avec ce qui est enfoui et le ramener au jour, comme une langue oubliée, comme des vestiges d'un monde tenu au seuil de celui qui est visible. Mais ce qui est dans l'ombre n'est pas pour autant oublié. L'écrivain est ainsi un explorateur des territoires perdus. Il est également un arpenteur de cette part de l'histoire actuelle que l'on ne veut pas voir ${ }^{(61)}$.

Une lecture attentive de plusieurs romans de Makine ${ }^{(62)}$, révèle un univers romanesque ancré dans l'histoire de son pays natal, la Russie du XXème siècle, du moins qu'on puisse dire : une toile historique. Un univers fictionnel morbide et une réalité sociopolitique et géographique qui s'interpénètrent, ce romancier talentueux nous donne à lire le tout dans un seul fil diégétique.

A travers le Testament français, une intrigue russe au goût de la littérature française, Makine ne relate pas l'histoire d'une collectivité russe, mais dans une perspective sélective il fait évoluer Aliocha, le héros, un russe tiraillé entre deux pays, deux langues et deux cultures, en le rattachant à des morceaux d'histoire à travers une écriture réaliste, brute et selon un point de vue, non documentaire, mais plutôt critique, et de témoignage ${ }^{(63)}$ mais avec beaucoup de nostalgie, amour inconditionnel et amertume, sentiments d'admiration et de rejet.

Il retrace, subjectivement, l'évolution et les changements de la Russie Soviétique durant les grandes périodes destructrices: la seconde guerre mondiale exterminatrice suivie de la guerre froide, des affrontements idéologiques, des rapports conflictuels avec plusieurs pays, des alliances et des désunions pleines de suspicions.

$\mathrm{Sa}$ fiction est une sorte «d'archivoir» pour «une histoire non encore, ou insuffisamment, déverrouillée ${ }^{(64)}$. Grace à la mémoire, Makine transfigure le réel à travers l'univers des rêves et de la fantaisie, un véritable roman d'initiation et d'apprentissage qui nous fait vivre un quotidien russe avec un ailleurs parisien. Conclusion 
«L'existence d'une réalité non verbale en dehors de l'univers de mots est indéniable. Toutefois, la croyance naïve en un contact ou une relation directe entre mots et référents est une illusion ${ }^{(65)}$

Après avoir procédé à une lecture intertextuelle de l'ensemble des œuvres du corpus, nous avons remarqué que "l'intertextualité est un phénomène qui oriente la lecture du texte et gouverne éventuellement l'interprétation» ${ }^{(66)}$.

Du point de vue de l'interprétation ${ }^{(67)}$, la recontextualisation sémantique et temporelle n'est pas sans risque, la reproposition de textes littéraires anciens, considérés comme une structure sémiotique qui a ses propres éléments interprétatifs, à travers des textes nouveaux n'est pas sans conséquences. Les traces intertextuelles présentes dans l'hypertexte guident la lecture intertextuelle aussi bien que la relation du texte à son interprétant, sa signification textuelle est déterminée par un objet intertextuel plus que le contexte, c'est pourquoi que le lecteur par souci d'économie interprétative ${ }^{(68)}$ pourrait mettre à l'écart certains éléments narratifs pour ne s'intéresser qu'à ceux qui sont dictées par l'hypotexte et orientent la lecture et le sens de l'interprétation dans un processus de coopération interprétative ${ }^{(69)}$.

C'est là que réside le génie de nos romanciers, ils ont emprunté ce chemin périlleux de la transposition textuelle, mais ils y sont bien préparés. Grâce aux techniques narratives mises en place par chaque écrivain qui ont, essentiellement, pour première fonction de distraire de plusieurs détails narratifs du premier texte en inventant un autre élément narratif propre au deuxième texte. En ce sens, la lecture intertextuelle permet une lecture de l'hypertexte et la compréhension du travail de l'écrivain, elle permet également de concevoir l'intertextualité (70) comme le moyen et le processus qui permet d'organiser la relation entre l'historcité ( la manière avec laquelle le social sature le texte) et la textualité, en faisant la relation au niveau textuel, de l'analyse discursive et l'analyse idéologique au sens bakhtinien ${ }^{(71)}$ de système d'idée, de croyances et de valeurs.

Donc l'évocation d'un ou de plusieurs intertextes par nos romanciers n'est pas innocente. Ces intertexte permettent de produire de nouvelles idées à travers d'anciens textes, ainsi, pour comprendre la vision idéologique dissimulée par le texte aboutit, l'intertextualité se limite désormais à une stratégie d'écriture qui permet la recontextualisation sémantique et temporelle du deuxième texte et c'est à l'interdiscursivité qu'il faut prêter plus d'attention, "pour renvoyer aux rapports plus large que le texte oral ou écrit entretint avec les énoncées (ou discours) enregistrés dans la culture correspondante et ordonnés selon une idéologie ${ }^{(72)}$. Balzac et La Petite tailleuse chinoise, Barbe Bleue et le testament français illustrent parfaitement le constat que les écrivains se sont servi de l'intertextualité comme "un principe actif de réalisation de l'intertdiscursivité au cœur du processus de structuration du texte ${ }^{(73)}$ 


\section{Balzac Et La Petite Tailleuse Chinoise, Barbe Bleue et le testament français :de l'intertextualité à le recontextualisation}

Les jeux intertextuels auxquels s'adonnent les romanciers s'inscrivent dans une véritable stratégie discursive et idéologique du romancier pour permettre une attitude réflexive et une lecture critique. Par ailleurs, l'intertextualité et l'intertdiscurssivité se complètent ainsi: la première est une relation de coprésence (facultative ou obligatoire) entre occurrences, la deuxième est une relation entre les types dont relèvent les occurrences.

Pour extérioriser son intention et son idéologie au moyen d'un encodage symbolique, l'écrivain se sert du jeu combinatoire et actualise des énonces qui ont été utilisés antérieurement et qui portent en eux des marques intertextuelles et interdiscursives. La rencontre de textes est productrice d'une écriture lettrée, d'une nouvelle langue plus expressive à visée idéologique, de nouvelles stratégies narratives et une nouvelle esthétique qui dotent le texte abouti d'une puissance réflexivité.

Dai Sijie, Amélie Nothomb, et André Makine ont concrétisé avec leurs romans, chacun dans son propre style, la rencontre deux orientations apparemment opposées de la littérature. D'un coté, comme discipline qui se sert d'elle-même et se préoccupe essentiellement du langage ; de la littérature comme, en même temps, nécessaire répétition et appropriation entre l'inédit et le déjà dit: « l'œuvre d'art, à l'époque moderne, semble retenir un rapport foncièrement nostalgique avec celles du passé,(...)si neuve soit elle, elle est un souvenir ${ }^{(74)}$. Et de l'autre coté, son aspect pragmatique qui exprime la condition humaine. Pourtant « faut-il attendre de la littérature qu'elle dégage une vérité humaine (...) des évènements ? ${ }^{(75)}$

\section{Bibliographie \\ Corpus}

1-Amélie Nothomb, Barbe Bleue, Albin Michel, 2012,180 p.

2-André Makine, Le testament français, Coll. Bleue, Mercure de France.1995, 320p.

3-Dai Sijie, Balzac et La Petite Tailleuse Chinoise, Paris, Gallimard, 2000,240p Ouvrages théoriques

1-Bruno Bettelheim, the uses of Enchantement, trad. psychanalyse des contes de fées, Robert Laffond, Paris, 1976

2-Florence Fix, Barbe Bleue et l'esthétique du secret de Charles Perrault à Amélie Nothomb, Hermann, 2014.

3-Gérard Genette, introduction à l'architexte, Paris, Seuil, 1979.

4-Gérard Genette, Palimpsestes, la littérature au second degré, Paris, Seuil, 1982.

5-Julia Kristeva, sémiotiké, recherche pour une sémanalyse, Seuil, coll. Points, Paris, 1969. 
6-Liu Jianmei , Révolution Plus Love: Histoire littéraire, corps de femmes et répétition thématique dans la fiction chinoise du vingtième siècle, presse universitaire de la Haye, 2003.

7-Lucien Dallenbache in Anna -Calire Gignoux, initiation à l'intertextualité, Ellipses, 2005.

8-Michael Riffaterre, «L'illusion référentielle », Columbia Review, 57 (2), 1978.

9-Michael Riffaterre, «La Trace de l'intertexte », La Pensée, $\mathrm{n}^{\circ}$ 215, 1980.

10-Michael Riffaterre, "l'intertexte inconnu », littérature n41, 1981.

11-Michel Le Bris et Jean Rouaud, Je est un autre, pour une identité-mode, Paris, Gallimard 2010.

11-Nathalie Heinich, Etats de femme. L'identité féminine dans la fiction occidentale, Paris, Gallimard, 1996p.180, cité dans, Florence Fix, Barbe Bleue et l'esthétique du secret de Charles Perrault à Amélie Nothomb, Hermann, 2014. 13-Paul Ricœur in A. Saudan et C. Villanueva dans, la littérature et la philosophie, Bréal, 2004.

14-Paul Ricœur, Soi même comme un autre, Paris, Seuil, 1990.

15-Roland Barthes, théorie du texte, Encyclopédie Universalis, t 15, Paris, 1973, pp.1013

16-Jean-Luc Domenach, «Chine : les balbutiements de l'histoire », Critique internationale 2004/3 (no 24).

17-Theirry Luarent, Andreï Makine et le bilan de l'URSS, Andreï Makine, Etudes réunies et présentées par Murielle Lucie Clément, périodique CRIN vol 53, 2009, pp88-92.

18-Théorie d'ensemble, textes réunis par Ph. Sollers, Paris, Seuil, 1971, p.75.

19-Tiphaine Samoyault, $l^{l}$ intertextualité mémoire de la littérature, Armand Colin,

Nathan, 2001.

20-Tzvetan Todorov, Mikhaïl Bakhtine, Le principe dialogique, Paris, Seuil., 1981 ,

21-Tzvetan Todorov, la notion de la littérature, Paris, Seuil, 1987.

22- Umberto Eco, lector in fabula, Grasset, 1979, trad.1985.

\section{Références électroniques}




\section{Balzac Et La Petite Tailleuse Chinoise, Barbe Bleue et le testament}

français :de l'intertextualité à le recontextualisation

1-Caroline Bouvier, La Barbe bleue, p.3 (http://www.rabeux.fr/medias/File/Dp\%20La\%20Barbe\%20bleue\%2011\%201 2.pdf )

tp://www.etudes-litteraires.com/la-princesse-de-cleves.php,consulté Le 12 septembre 2016

2-Roland BARTHES, «Texte (Théorie du) », Encyclopedia Universalis, 1973 ; cité

danshttp://www.fabula.org/atelier.php?Intertextualit\%26eacute\%3B et interdi scursivit\%26eacute\%3B.

3-Zineb Ali Benali, «Le roman, cet archiviste de l'histoire», Insaniyat / إنسانيات [En ligne], 21 | 2003, mis en ligne le 30 septembre 2012, consulté le 21 mai2016. URL: http://insaniyat.revues.org/7320 ; DOI : 10.4000/insaniyat.7320

4--http://www.leparisien.fr/flash-actualite-culture/le-retour-en-russie-d-andreimakine-le-plus-russe-des-ecrivains-francais-27-08-2016-6073331.php

5-«Les lieux du discours littéraire », Lieux communs. Topoï, stéréotypes, clichés, dir. Christian Plantin, Paris, éd. Kimé, 1993, p. 92-100. Anne-Claire Gignoux, "De l'intertextualité à l'écriture», Cahiers de Narratologie [En ligne], 13 | 2006, mis en ligne le 01 septembre 2006, consulté le 08 mars 2016. URL : http:// narratologie.revues.org/329 ; DOI : 10.4000/narratologie.329

\section{Note :}

(1) - Dai Sijie, Balzac et La Petite Tailleuse chinoise, Paris, Gallimard, 2000,240p.

(2) - Amélie Nothomb, Barbe Bleue, Albin Michel, 2012,180 p.

(3) - André Makine, Le testament français, Coll. Bleue, Mercure de

France.1995, 320p.

(4) - T. Samoyault, intertextualité, mémoire de la littérature, Armand Colin, 2001, p.50

(5) - Dans son ouvrage problème de la poétique de Dostoïevski réédité pour la deuxième fois en 1965.

(6) - M. Bakhtine, "du discours romanesque » in Esthétique et théorie du roman, trad. Par Daria Olivier, Paris, Gallimard, 1978, p 155.

(7) - Kristeva forge en Français le terme de « l'intertextualité », dans son article " le mot, le dialogisme et le roman » (qui date de 1966 mais publié en 1969)

(8) - J.Kristeva, «Le mot, le dialogue et le roman », Sémiotikè, recherche pour une sémanalyse, Seuil, coll. Tel Quel, 1969, p.83.

(9) - J. Kristeva, sémiotiké, recherche pour une sémanalyse, Seuil, coll. Points, Paris, 1969, pp.84-85 
(10) - T. Todorov, Mikhaïl Bakhtine, Le principe dialogique, Paris, Seuil., 1981, p.8

(11) - M. Riffaterre, « l'intertexte inconnu », littérature n41,1981,p.p.4-5

(12) - M. Riffaterre, «La Trace de l'intertexte », La Pensée, n² 215, 1980, p.4

(13) - G. Genette, palimpseste, la littérature au second degré, Paris, Seuil, 1982, p7

(14) - G. Genette, introduction à l'architexte, Paris, Seuil, 1979.p 87.

(15) - R. BARTHES, «Texte (Théorie du)», Encyclopédie Universalis, 1973 ; cité dans

http://www.fabula.org/atelier.php?Intertextualit\%26eacute\%3B et interdiscurs ivit\%26eacute\%3B , consulté le 12 juin 2016

(16) - R. Barthes, théorie du texte, Encyclopédie Universalis, t 15, Paris, 1973, pp.1013-7.

(17) - J. Sclanger, La mémoire des œuvres, Nathan, 1992, p.114

(18) - T.Todorov, la notion de la littérature, Paris, Seuil, 1987.p 29

(19) - P. Ricœur in A. Saudan et C. Villanueva dans, la littérature et la philosophie, Bréal, 2004, p.35.

(20) - Ibid., p.37

(21) - Dai Sijie, Balzac et La petite Tailleuse chinoise, Gallimard, 2000, p.54

(22) - Ibid., p.126

(23) - Ibid., p 127

(24) - Ibid., p 71

(25) - L. Dallenbache in Anna-Calire Gignoux, initiation à l'intertextualité,

Ellipses, 2005, p.43.

(26) - D. Sijie, op.cit., p137

(27) - Ibid. p.158.

(28) - Appelée également la littérature de réforme et de reportage, tire son nom : elle est initiée par Liu Xinwu et tire son nom de sa deuxième nouvelle La cicatricepubliée le 11 août 1978 dans le 'Wenhui bao') de Shanghai, tirée à 150000 exemplaires.

(29) - J.L Domenach, "Chine : les balbutiements de l'histoire », Critique internationale 2004/3 (no 24), p. 82.

(30) - Liu Jianmei, Révolution Plus Love: Histoire littéraire, corps de femmes et répétition thématique dans la fiction chinoise du vingtième siècle, presse universitaire de la Haye, 2003, p.277.

(31) - Ibid.

(32) - D.Sijie, Op.cit., p.229.

(33) - T. Samoyault, $l^{l}$ 'intertextualité mémoire de la littérature, Armand Colin, Nathan, 2001, p.55.

(34) - "Les lieux du discours littéraire », Lieux communs. Topoï, stéréotypes, clichés, dir. Christian Plantin, Paris, éd. Kimé, 1993, p. 92-100. Anne-Claire Gignoux, "De l'intertextualité à l'écriture», Cahiers de Narratologie [En 


\section{Balzac Et La Petite Tailleuse Chinoise, Barbe Bleue et le testament français :de l'intertextualité à le recontextualisation}

ligne], 13 | 2006, mis en ligne le 01 septembre 2006, consulté le 08 mars 2016. URL : http:// narratologie.revues.org/329 ; DOI : 10.4000/narratologie.329

(35) - C. Bouvier, La Barbe Bleue, p. 3

(http://www.rabeux.fr/medias/File/Dp\%20La\%20Barbe\%20bleue\%2011\%201 $\underline{2 . p d f)}$

(36) - Amélie Nothomb, Barbe Bleue, Albin Michel, 2012, p.97.

(37) - Ibid., p 93.

(38) - Ibid. .p 156.

(39) - Ibid. p.23.

(40) - T. Samoyault, op.cit, p.54

(41) - A. Nothomb, op.cit., p30.

(42) - Ch. Perrault, Peau D'âne, les Coignard, Paris, 1694.

(43) - A. Nothomb, Barbe Bleue, Albin Michel, 2012, p.111.

(44) - M.M.de La Fayette, la princesse de Clèves, Claude Barbin, Paris, 1678.

(45) - http://www.etudes-litteraires.com/la-princesse-de-cleves.php,consulté Le 12 septembre 2016

(46) - A. Notomb, op.cit., p.111.

(47) - G. Flaubert, Correspondance, Gallimard, »Bibliothèque de la Pléiade », 1973, I, p.302 ; cité dans T. Samoyault , op.cit.p.57.

(48) - F. Fix, Barbe Bleue et l'esthétique du secret, de Charles Perrault à

Amélie Nothomb, Hermann, 2014

(49) - B. Bettelheim, the uses of Enchantement, trad. psychanalyse des contes de fées, Robert Laffond, Paris, 1976

(50) - F. Fix, Barbe Bleue et l'esthétique du secret de Charles Perrault à Amélie Nothomb, Hermann, 2014,p.23.

(51) - Ibid, la quatrième de couverture.

(52) - B. Bettelheim, op.cit.

(53) - G. Genette, op.cit. , p.17.

(54) - N. Heinich, Etats de femme. L'identité féminine dans la fiction occidentale, Paris, Gallimard, 1996p.180, cité dans, F. Fix, op.cit, p.53.

(55) - M. Le Bris et J. Rouaud, Je est un autre, pour une identité-mode, Paris, Gallimard 2010, p.8.

(56) - P. Ricœur, Soi même comme un autre, Paris, Seuil, 1990, p.191.

(57) - http://www.leparisien.fr/flash-actualite-culture/le-retour-en-russie-dandrei-makine-le-plus-russe-des-ecrivains-francais-27-08-2016-6073331.php

(58) - A. Makine, Le testament français, Coll. Bleue, Mercure de France.1995, p.56

(59) - Ibid,p. 56

(60) - Ibid, p. 46

(61) - Ibid. 
(62) - T. Luarent, «Andreï Makine et le bilan de l'URSS », Andreï Makine, Etudes réunies et présentées par Murielle Lucie Clément, périodique CRIN vol 53, 2009, pp88-92.

(63) - Il était un ex-réfugié politique.

(64) - Z. Ali Benali, «Le roman, cet archiviste de l'histoire », op.cit.

(65) - M. Riffaterre , «L'illusion référentielle », Columbia Review, 57 (2),

1978 .p.92

(66) - M. Riffaterre, l'intertexte inconnu 1981, p4-5.et 6

(67) - Ibid.

(68) - U.Eco, lector in fabula, Grasset, 1979, trad.1985.

(69) - Ibid.

(70) - G. Genette, palimpseste, la littérature au second degré, Paris, Seuil, 1982, p97

(71) - C.Serge «Intertestualtà et interdisousività nel romanzo e nella poesia » tatro e romanzo. Turin, Einaudri, 1984, en particulier, p.106 ; cité dans, Eric Le Calvez, Marie-Claude Canoua-Green, texte(s) et intertexte(s), Rodopi, 1997, p28

(72) - ibid, p24.

(73) - S. Vultur « situer l'intertextualité » cahiers Roumains d'études littéraires, VIII, 3, 1981, p.35; cité dans, op.cit.p.25

(74) - M. schneider, voleurs de mots, essai sur le plagiat, la psychanalyse et la pensée, Paris Gallimard, 1985,p53

(75) - C. Herzefeld le peuple et la mer, in PAUL Jean Marie( sous la dir), le peuple, mythe et réalité, presse universitaire de Rennes, Rennes Cedex, 2007,pp.123,124. 